\title{
AN ADAPTIVE IDENTIFICATION METHOD BASED ON THE MODULATING FUNCTIONS TECHNIQUE AND EXACT STATE OBSERVERS FOR MODELING AND SIMULATION OF A NONLINEAR MISO GLASS MELTING PROCESS
}

\author{
WITOLD BYRSKI $^{a, *}$, MichA£ DRAPAŁA $^{a, c}$, JĘDRZEJ BYRSKI $^{b}$ \\ ${ }^{a}$ Department of Automatic Control and Robotics \\ AGH University of Science and Technology, Al. Mickiewicza 30, 30-059 Kraków, Poland \\ e-mail: \{wby, marapala\} @agh.edu.pl \\ ${ }^{b}$ Department of Applied Computer Science \\ AGH University of Science and Technology, Al. Mickiewicza 30, 30-059 Kraków, Poland \\ e-mail: jbyrski@agh.edu.pl \\ ${ }^{c}$ Techglass Sp. z o.o. \\ ul. Zygmunta Starego 124, 30-198 Kraków, Poland \\ e-mail: michal.drapala@techglass.pl
}

\begin{abstract}
The paper presents new concepts of the identification method based on modulating functions and exact state observers with its application for identification of a real continuous-time industrial process. The method enables transformation of a system of differential equations into an algebraic one with the same parameters. Then, these parameters can be estimated using the least-squares approach. The main problem is the nonlinearity of the MISO process and its noticeable transport delays. It requires specific modifications to be introduced into the basic identification algorithm. The main goal of the method is to obtain on-line a temporary linear model of the process around the selected operating point, because fast methods for tuning PID controller parameters for such a model are well known. Hence, a special adaptive identification approach with a moving window is proposed, which involves using on-line registered input and output process data. An optimal identification method for a MISO model assuming decomposition to many inner SISO systems is presented. Additionally, a special version of the modulating functions method, in which both model parameters and unknown delays are identified, is tested on real data sets collected from a glass melting installation.
\end{abstract}

Keywords: continuous systems, modulating functions, system identification, exact state observer, glass forehearths.

\section{Introduction}

One of the still difficult problems in real-time control of complex and distributed multi-input single-output (MISO) industrial processes is not only the proper choice of the vector of reference values (set points) for local PID controllers, which control single-input single-output (SISO) subsystems, but also the proper choice of PID controller parameters. This is especially crucial if the operating points are often changed. The rules of fast PID controller tuning are well known, but they assume the knowledge of the linear SISO model parameters for

* Corresponding author the corresponding subprocess. Hence, to apply these rules, the supervisory control level should have the most recent linear models of local subprocesseses. In some domains, the continuous methods dominate over the classical discrete approach. Hence, a continuous methodology to on-line passive (without step response) identification of a continuous industrial process is used in this paper.

The presented identification approach uses the modulating functions method (MFM) and is based on on-line building a continuous linear dynamic model for the nonlinear MISO industrial process, which works in a standard closed-loop control system with local PID 
controllers. In the MISO system only the system inputs and the output are measured, therefore, the output of each SISO subprocess is unknown. Hence, for the identification of each SISO model a special procedure has to be elaborated. The identification procedure is performed for consecutive time intervals (the moving window of a chosen width) and executed only on the intervals in which the simulated output of the MISO model and the real system output differ from each other. If the simulated and real outputs are similar (the error value is less than a predefined threshold), the identification procedure is not performed on this interval (the last obtained model is valid). A detailed description of this procedure and adopted parameter values are presented in the experimental part of the paper.

If for the current interval, the system output fulfills requirements of the operating point and simultaneously the model predicts this output correctly, then the algorithm does not change anything. If, however, the dynamic changes in the real process output (and, simultaneously, the corresponding MISO model output) do not fulfill requirements of the operating point in the current interval, then the actual linear SISO models can be treated as a basis for a possible correction of PID controller parameters (PID adaptive tuning). Tuned parameters are applied to the controllers in the next time interval.

This paper focuses only on the identification aspects. The procedure of PID parameter tuning is not discussed. As was already mentioned, if the current model output differs from the measured output, then the identification procedure has to be executed in this interval. The model parameters are updated for the next intervals. The controller parameters can be tuned based on the new model. The identification procedure can be repeated in the last interval many times for the single SISO models. Such an adaptive correction procedure enables obtaining optimal parameters of the MISO model.

The lack of an effective solution for the problem of on-line PID controller tuning and the need to improve the computer control system in the real glass melting plant was the main motivation of the described research. A new contribution to the theory and practice of control systems is the use of a nonstandard optimal identification method (with modulating functions), which up to now was used mainly for SISO models. The extension of this method to its adaptive version and its use for MISO systems, as well as for systems with multiple time-delay inputs, is a key novelty of this paper. Another quite new idea is the decomposition of the optimization algorithm into two stages with the use of two different performance indexes (the equation error for local solutions of SISO models and the output error for global MISO solution). The described algorithms are tested on real process data sets collected in a glassworks, which produces glass containers.

\section{Basics of the MFM}

In the standard $n$-th order input-output differential equation

$$
\sum_{i=0}^{n} a_{i} y^{(i)}(t)=\sum_{j=0}^{m} b_{j} u^{(j)}(t)
$$

of the continuous SISO linear time invariant (LTI) model, $n+1$ parameters $a_{i}$ associated with derivatives of the output signal $y(t)$ as well as $m+1$ parameters $b_{i}$ associated with the derivatives of the input signal $u(t)$ should be identified. The most common situation is assumed, which mostly occurs in industrial applications, that the signals $y(t)$ and $u(t)$ are measured, but their derivatives are not. For parameter identification in this type of continuous systems, various approaches can be used. Basically, the results of $N$ experiments must be obtained, where in general $N \gg n+m+2$. An overview of continuous system identification methods can be found in the work by Rao and Unbehauen (2006).

One of these methods is the idea of the MFM, which was originally developed by Shinbrot (1957) for linear and nonlinear dynamic systems. Its main advantages are the following:

- no need to discretize a continuous time model,

- no need to know model input and output signals derivatives,

- elimination of the influence of the initial conditions,

- a finite time interval used in the identification process.

Many authors extended this method by some modifications and generalizations to make it suitable for certain problems. Application for MIMO continuous systems was proposed by Byrski and Kubiński (1997). A generalization for fractional differential equations was made by Janiczek (2010) as well as Asiri and Laleg-Kirati (2017). Algorithms for models with unknown delays and for Hammerstein models can be found in works of Balestrino et al. (2000a; 2000b). MFM related approaches were also used for state observation. Examples of such applications can be found in the works of Byrski and Byrski (2016) or Jouffroy and Reger (2015).

The MFM is based on the use of the inner product of functions given by an integral formula and the rule of integration by parts. Using it on the left-hand side of (1), we can see that

$$
a_{i} \int_{0}^{h} y^{(i)}(\tau) \phi(\tau) \mathrm{d} \tau=a_{i}(-1)^{i} \int_{0}^{h} y(\tau) \phi^{(i)}(\tau) \mathrm{d} \tau
$$

for $i \in\{0, \ldots, n\}$. This formula is valid under the assumptions that the values of functions $y(t), \phi(t)$ and $\phi^{(i)}(t)$ are in the interval $[0, h]$ and, additionally, $\phi^{(i)}(\tau)$ 
fulfills special boundary conditions: $\phi(0)=\ldots=$ $\phi^{(n-1)}(0)=\phi^{(n)}(0)=0$ and $\phi(h)=\ldots=$ $\phi^{(n-1)}(h)=\phi^{(n)}(h)=0$. The functions $y^{(i)}(\tau)$ are unknown. Three different approaches are used in the MFM.

In the first approach, one should repeat for (1) the above integral formula $N$ times in the same interval $[0, h]$ for $N$ different modulating functions $\phi_{k}(\tau)$, where $k=$ $1, \ldots, N$. The signal $y(\tau)$ should be given within the same time interval $[0, h]$. The modulating function $\phi_{k}(\tau)$ should be used for each $i$-th and $j$-th term for the whole equation

$$
\begin{aligned}
& a_{i} \int_{0}^{h} y^{(i)}(\tau) \phi_{k}(\tau) \mathrm{d} \tau \\
&=a_{i}(-1)^{i} \int_{0}^{h} y(\tau) \phi_{k}^{(i)}(\tau) \mathrm{d} \tau
\end{aligned}
$$

It will generate the set of $N$ independent algebraic linear equations representing effects of filtering $y(t)$ on $[0, h]$ by the use of $N$ different finite impulse response (FIR) filters. It allows us to obtain the solution of the identification problem on the interval $[0, h]$ based on the output error method (OEM) and minimization of the sum of $N$ square errors, utilizing the least-squares method. Examples of this methodology can be found in works of Jouffroy and Reger (2015) and Cieza et al. (2014).

In the second approach, one can use only one modulating function $\phi(\tau)$, given on the interval $[0, h]$, but used for $N$ different fragments of the signal $y(\tau)$ which was measured within $N$ different time intervals $\left[t_{k-h}, t_{k}\right]$ of the same width $h$, where $k=1, \ldots, N$, within the whole identification interval $T_{I D}, t_{N}<T_{I D}$. The equation is in the form

$$
\begin{aligned}
a_{i} \int_{t_{k}-h}^{t_{k}} y^{(i)}(\tau) & \phi(\tau) \mathrm{d} \tau \\
& =a_{i}(-1)^{i} \int_{t_{k}-h}^{t_{k}} y(\tau) \phi^{(i)}(\tau) \mathrm{d} \tau
\end{aligned}
$$

The signal $y(t)$ is assumed to be persistently exciting of a sufficient order. Then it will generate a set of $N$ independent linear equations representing effects of filtering $y(t)$ with the use of one FIR filter in different time intervals. It allows finding the solution to the parameter identification problem for the whole interval $\left[0, T_{I D}\right]$ based on OEM and minimization of the sum of $N$ square errors, which can be expressed by the formula for the optimal parameters, which follows from the least-squares method. The result of such identification can give satisfactory results if the system is stationary (has time-invariant parameters). If the system is non stationary, then this approach will yield a result, which will represent the average parameters values $\theta$ for the whole interval $\left[0, T_{I D}\right]$. The described method was applied by Balestrino et al. (2000a; 2000b).

In the above two approaches, the method of $N$ repeated experiments enables using the sum of $N$ square errors. In these methods calculations in the continuous time domain are also applied (integrals).

In the third approach, presented by Byrski and Fuksa (1995), one can use a fully continuous methodology for parameter identification of the continuous system with the use of convolution transformation and only one modulating function $\phi(\tau)$, which has compact support $[0, h]$ - it is nonzero inside this interval and is zero outside this interval. This function forms a continuous moving convoluting window of width $h$ within the interval $[t-$ $h, t], \quad \forall t \in\left[h, T_{I D}\right] . \quad$ In the interval $[t-h, t]$ the convolution function is convoluted with each $i$-th term of the differential equation

$$
\begin{aligned}
& a_{i} \int_{-\infty}^{\infty} y^{(i)}(\tau) \phi(t-\tau) \mathrm{d} \tau \\
& \quad=a_{i} \int_{t-h}^{t} y(\tau) \phi^{(i)}(t-\tau) \mathrm{d} \tau \\
& \quad=a_{i} \int_{0}^{h} y(t-\tau) \phi^{(i)}(\tau) \mathrm{d} \tau \stackrel{\text { def }}{=} a_{i} y_{i}(t) .
\end{aligned}
$$

This process will generate new continuous functions $y_{i}(t)$ and $u_{i}(t)$ from Eqn. (1) within the whole identification interval $t \in\left[h, T_{I D}\right]$, which can be interpreted as an infinite number of computational experiments performed continuously, within each interval $[t-h, t]$ for each moment of time $t \in\left[h, T_{I D}\right]$. Based on the equation error method (EEM) and these functions, instead of a finite sum of $N$ square errors, one can calculate the values of the new square error integral in the whole identification interval $\left[h, T_{I D}\right]$. This second integral represents the inner products of these functions and finally enables calculation of the real Gram matrix $\mathbf{G}\left(T_{I D}\right)$. Using its inverse $\mathbf{G}^{-1}\left(T_{I D}\right)$ and a modified least-squares method, the parameters $\boldsymbol{\theta}\left(T_{I D}\right)$ can be found. In this approach, the on-line continuous version of parameter identification for $t>T_{I D}$ is also possible. To this end, the moving identification window within the current time interval $[t-$ $\left.T_{I D}+h, t\right]$ for $t>T_{I D}$ can be used based on continuous calculation of the integral inner products and continuous evolution of the Gram matrix $\mathbf{G}(t)$. If the system is nonstationary, then it allows tracking the evolution of time-variant parameters $\boldsymbol{\theta}(t)$. If the system is stationary, it will give the same constant value $\boldsymbol{\theta}(t)=\boldsymbol{\theta}\left(T_{I D}\right)$. It is very useful for on-line identification of continuous LTI systems with different constraints for parameters. New modifications of this idea can be found in the work of Byrski and Byrski (2012). In this paper, the third approach presented above will be used. 


\section{MFM used for the MISO system}

Starting from this section, the index $k$ will be used for numbering SISO subsystems forming the MISO system, in contrast to the previous section, where it was used as the number of the subsequent experiment. The differential equation representing the linear MISO system with $K$ inputs and single output can be expressed as

$$
\begin{aligned}
\sum_{i=0}^{n} a_{i} y^{(i)}(t) & =\sum_{k=1}^{K} \sum_{j=0}^{m_{k}} b_{k j} u_{k}^{(j)}(t) \\
& =\sum_{j=0}^{m_{1}} b_{1 j} u_{1}^{(j)}(t)+\cdots+\sum_{j=0}^{m_{K}} b_{K j} u_{K}^{(j)}(t) .
\end{aligned}
$$

Functions $y^{(i)}, u_{1}^{(j)}, \ldots, u_{K}^{(j)}$ are the derivatives of the inputs and the output given on the interval $\left[t_{0}, T_{I D}\right]$. There are $n$ output derivatives and $m_{k}$ derivatives for the $k$-th input, where $m_{k} \leq n, \quad \forall k=1, \ldots, K$. The corresponding diagram is presented in Fig.1.

Equation (6) represents the MISO system with an assumed special feature, i.e., that all SISO transfer functions, which form the MISO system, have their common denominator. Apart from this structure, in the identification algorithm presented in this paper also the differential equation

$$
\sum_{i=0}^{n} a_{k i} y_{k}^{(i)}(t)=\sum_{j=0}^{m_{k}} b_{k j} u_{k}^{(j)}(t)
$$

for each individual $k$-th SISO system forming the MISO system

$$
\sum_{k=1}^{K} y_{k}(t)=y(t)
$$

will be used. In this case, individual SISO systems may have different denominators.

Equation (6) can be transformed into an algebraic one after performing convolutions with the modulating

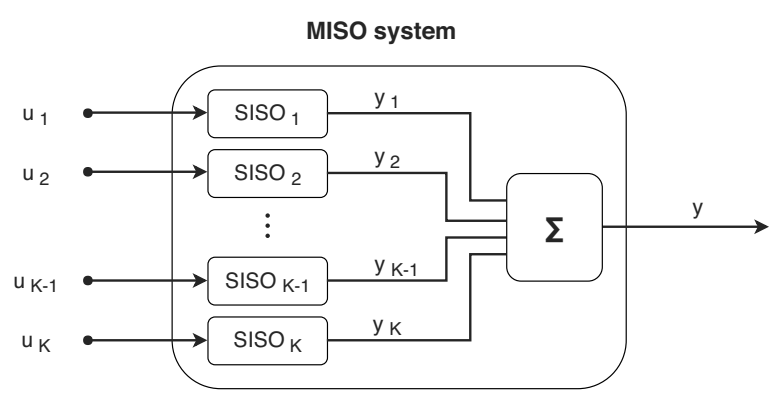

Fig. 1. MISO model diagram. function $\phi$ using the property

$$
\begin{aligned}
y_{i}(t) & =\left[y^{(i)} * \phi\right](t)=\left[y * \phi^{(i)}\right](t), \\
u_{i}(t) & =\left[u^{(i)} * \phi\right](t)=\left[u * \phi^{(i)}\right](t) .
\end{aligned}
$$

The new equation

$$
\begin{aligned}
& \sum_{i=0}^{n} a_{i} y_{i}(t) \\
& \quad=\sum_{j=0}^{m_{1}} b_{1 j} u_{1 j}(t)+\cdots+\sum_{j=0}^{m_{K}} b_{k j} u_{K j}(t)+\epsilon(t)
\end{aligned}
$$

has the same vector of $n+m_{1}+\cdots+m_{K}+K+1$ unknown parameters $a_{i}$ and $b_{1 j}, \ldots, b_{K j}$.

Remark 1. Unless otherwise stated, in this section the lower indices of $y$ will be used for functions obtained after the convolution transformation, like in (10). They are not the same as the original functions $y_{k}^{(i)}(t)$ in (7) and $y_{k}(t)$ in (8).

The modulating function $\phi$ should be defined on the whole space $\mathbb{R}$ and meet specified conditions:

- $\phi$ is supposed to be nonzero in the interval $[0, h]$ and zero outside this interval, i.e., it has a compact support of width $h$ (closed and bounded); for $t_{0}=$ 0 , the identification interval should be $T_{I D}>2 h$ (however, it is not necessary and, formally, it is enough if $T_{I D}>h$ );

- $\phi \in C^{n-1}[0, h]$ is $n-1$ times differentiable;

- $\phi^{(i)}(0)=\phi^{(i)}(h)=0$ for $i=0, \ldots, n-1$.

- $y * \phi=0 \Rightarrow y=0$ on the interval $\left[t_{0}+h, T_{I D}\right]$.

In this paper, the Loeb-Cahen functions

$$
\phi(t)=t^{N}(h-t)^{M}
$$

will be used. Other authors proposed different types of functions. Especially spline modulating functions have strong theoretical basis (Maletinsky, 1979; Preisig and Rippin, 1993). Efficient identification algorithms using sinusoidal modulating functions related to the fast Fourier transform can be found in the works of Pearson et al. (1994) or Co and Ydstie (1990). The transformed functions have the form

$$
y_{i}=y(t) * \phi^{(i)}(t)=\int_{t-h}^{t} y(\tau) \phi^{(i)}(t-\tau) \mathrm{d} \tau .
$$

Due to the signal noise and the equation error, in (10) there is the term $\epsilon(t)$ representing the difference between the convoluted signals for system inputs and output. The discussion of disturbances and their influence on the equation error (EE) was made by Byrski and Byrski (2012). 
The square error value $\epsilon(t)$ can be treated as a performance index for the MISO model (6) as well as for the selected SISO models (7). Denoting by $\boldsymbol{\theta}$ the vector of the parameters of the model (6) and by $\boldsymbol{c}(t)$ the modulated measurements vector, the equation error can be obtained as

$$
\begin{aligned}
& \epsilon(t)=\boldsymbol{c}^{T}(t) \boldsymbol{\theta} \\
& =\left[y_{0}(t), \ldots, y_{n}(t),-u_{10}(t), \ldots,\right. \\
& \left.\quad-u_{1 m_{1}}, \ldots,-u_{K 0}, \ldots,-u_{K m_{K}}(t)\right]\left[\begin{array}{c}
\boldsymbol{a} \\
\boldsymbol{b}_{1} \\
\vdots \\
\boldsymbol{b}_{K}
\end{array}\right],
\end{aligned}
$$

where $\boldsymbol{a}, \boldsymbol{b}_{1}, \ldots, \boldsymbol{b}_{K}$ are column vectors of suitable dimensions $n+1, m_{1}+1, \ldots, m_{K}+1, \quad \boldsymbol{\theta} \in$ $\mathbb{R}^{n+m_{1}+\cdots+m_{K}+K+1}$.

The minimization problem can be formulated in $L^{2}\left[t_{0}+h, T_{I D}\right]$ as

$$
\min _{\theta} J^{2}=\min \|\epsilon(t)\|_{L^{2}\left[t_{0}+h, T\right]}^{2}=\min \left\|\boldsymbol{c}(t)^{T} \boldsymbol{\theta}\right\|_{L^{2}}^{2} .
$$

In order to avoid a trivial solution, the following linear constraint is assumed:

$$
\boldsymbol{\eta}^{T}\left[\begin{array}{c}
\boldsymbol{a} \\
\boldsymbol{b}_{1} \\
\vdots \\
\boldsymbol{b}_{K}
\end{array}\right]=\boldsymbol{\eta}^{T} \boldsymbol{\theta}=1
$$

where $\boldsymbol{\eta} \in \mathbb{R}^{n+m_{1}+\cdots+m_{K}+K+1}$ is an arbitrarily chosen vector and $\boldsymbol{\eta} \neq 0$. The norm in (14) can be written down as the inner product in $L^{2}$,

$$
\begin{aligned}
J^{2} & =\left\langle\boldsymbol{c}^{T}(t) \boldsymbol{\theta}, \boldsymbol{c}^{T}(t) \boldsymbol{\theta}\right\rangle_{L^{2}} \\
& =\boldsymbol{\theta}^{T}\left\langle\boldsymbol{c}(t), \boldsymbol{c}^{T}(t)\right\rangle \boldsymbol{\theta} \\
& =\boldsymbol{\theta}^{T} \mathbf{G} \boldsymbol{\theta} .
\end{aligned}
$$

Remark 2. In the paper the special non-italic font was used for the Gram matrix $\mathbf{G}$ in contrast to other expressions denoted by $G$.

The square real Gram matrix $G$ (18) in the above quadratic form (16) is created by the inner products in $L^{2}$ of $\boldsymbol{c}(t)$ elements, where

$$
\left\langle y_{i}, u_{j}\right\rangle=\int_{t_{0}+h}^{T_{I D}} y_{i}(\tau) u_{j}(\tau) \mathrm{d} \tau .
$$

The optimal solution for the given linear constraint $\boldsymbol{\eta}$ can be found using the Lagrange multiplier technique:

$$
L=\boldsymbol{\theta}^{T} \mathbf{G} \boldsymbol{\theta}+\lambda\left(\boldsymbol{\eta}^{T} \boldsymbol{\theta}-1\right) .
$$

Based on necessary optimality conditions, the solution can be obtained in the form

$$
\boldsymbol{\theta}^{o}=\frac{\mathbf{G}^{-1} \boldsymbol{\eta}}{\boldsymbol{\eta}^{T} \mathbf{G}^{-1} \boldsymbol{\eta}}
$$

Substituting (20) into (16), another formula for the minimal value of the identification performance index can be obtained as

$$
J=\frac{1}{\sqrt{\boldsymbol{\eta}^{T} \mathbf{G}^{-1} \boldsymbol{\eta}}} .
$$

Byrski and Byrski (2012) thoroughly discuss selection of the vector $\boldsymbol{\eta}$. Taking this vector from a unit ball, it appears that the best $\boldsymbol{\eta}$, which additionally minimizes the performance index 21), is the eigenvector of the Gram matrix $\mathbf{G}$ (18), which corresponds to the minimal eigenvalue of $\mathbf{G}$. Such a direction of the subspace for the parameters constraint guarantees the minimum of the mean square equation error, what has the interpretation of the best parameters estimate under the Gaussian white noise existence in measurements. A more thorough discussion of the disturbance presence and its influence on the EE was made by Byrski and Byrski (2012). However, it turns out that in the case of nonlinear system identification better results can be obtained by decomposition of the MISO system to $K$ SISO separate subsystems. Each of these $\mathrm{SISO}_{k}$ models can be identified independently. Assuming a new performance index, defined as the squared difference between the real system output and the simulated MISO model output (OEM), another constraint vector $\boldsymbol{\eta}_{k}$ for the $k$-th SISO subsystem should be used. In the proposed algorithm, the resulting MISO model is identified with the use of the Gauss-Seidel-like method and the OEM as the main performance index, while in each direction (for a single SISO model) the EEM is used as the method for local identification. The constraint vector $\boldsymbol{\eta}_{k}$ can be interpreted as the directional variable in each step of the method. For each $\boldsymbol{\eta}_{k}$ the formula (20) will provide the local $\mathrm{SISO}_{k}$ model what enables simulation of the single output $y_{k}$.

3.1. Identification of the MISO system with delays. Many physical systems can be described as input-delay systems. The original continuous version of the applied MFM described by Byrski and Fuksa (1995) was intended for systems without delays. Rao and Sivakumar (1979), suggested a method of identifying the system delay by minimizing a cost function whose value depends on the difference between the process and model outputs. An interesting method of identifying both the linear system parameters and delay was proposed by Kozłowski and Kowalczuk (2015). The algorithm presented by Balestrino et al. (2000b) can be used for a certain class of systems in the case of single and multiple delays between inputs and outputs and involves introducing additional time-shifted 


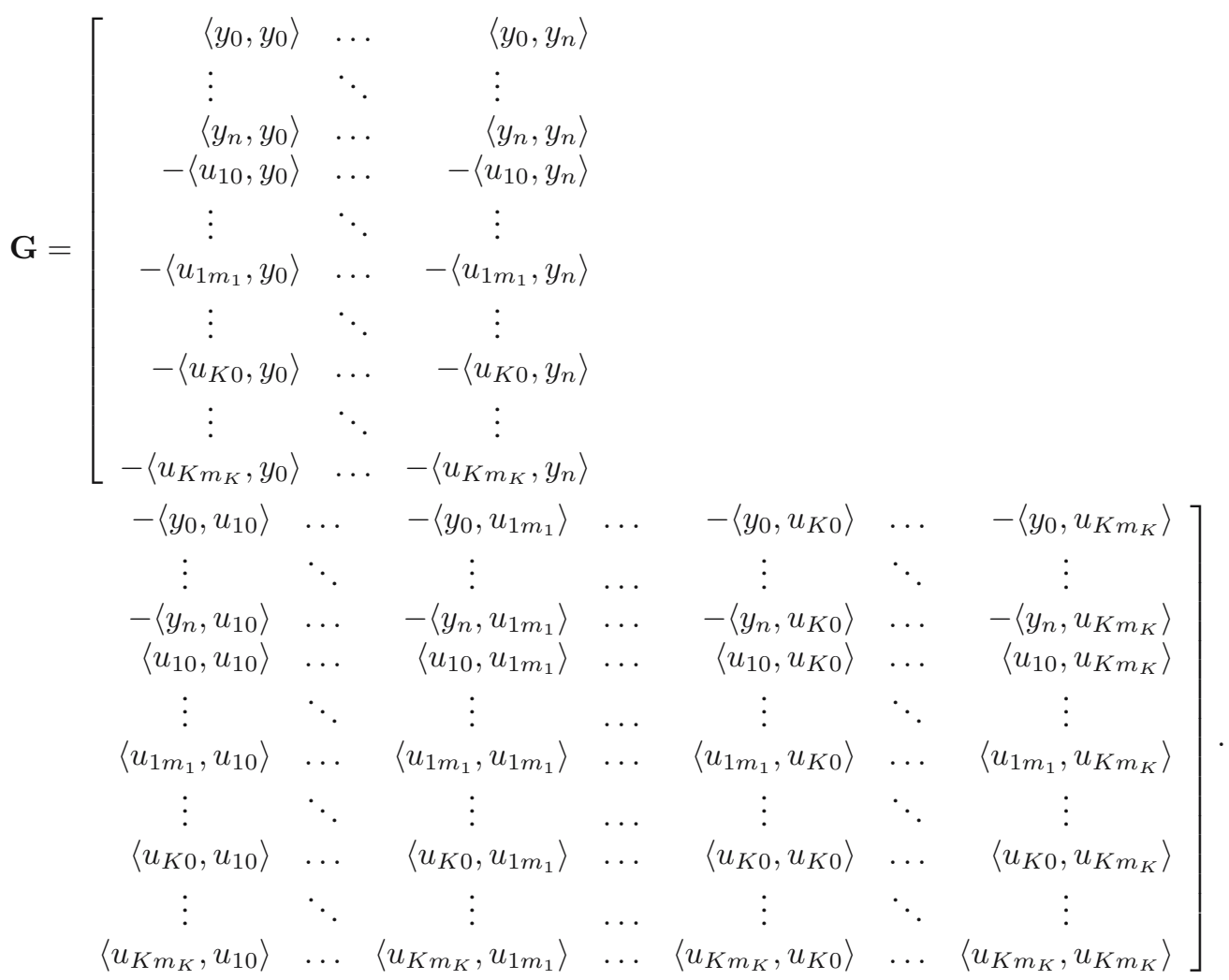

signals for the system inputs. A modified form of the MISO model (6) is adopted. Instead of many derivatives of each control signal $u_{k}^{(j)}(t)$ for the $k$-th SISO model, signal $u_{k}(t)$ itself is included. The multi-delay structure presented in Fig. 2 is assumed. This version of the MISO system with $K$ inputs has the form

$$
\sum_{i=0}^{n} a_{i} y^{(i)}(t)=\sum_{k=1}^{K} \sum_{q=0}^{Q_{k}-1} b_{k q} u_{k q}(t)
$$

where $u_{k q}(t)=u_{k}\left(t-\left(T_{k}+q \tau_{k}\right)\right)$. Hence, finally in this paper, MISO systems with an identified transfer function numerator of zero degree are considered: $m_{k}=$ $0, \quad \forall k=1, \ldots, K$.

System (22) can now be transformed using modulating functions into the form

$$
\sum_{i=0}^{n} a_{i} y_{i}(t)=\widetilde{\boldsymbol{u}}(t)^{T} \widetilde{\boldsymbol{b}}
$$

In the vector of modulated input signals $\widetilde{\boldsymbol{u}}(t)$, there are $Q_{k}$ time-shifted signals for each $k$-th input, where $q=1, \ldots, Q_{k}-1$. Starting from the second, the $q$-th signal is shifted by the constant time interval $\tau_{k}$ with respect to the previous vector element. The first signal corresponds to the system input $T_{k 0}$ seconds ago. Vector $\widetilde{\boldsymbol{u}}(t)$ has the form

$$
\widetilde{\boldsymbol{u}}(t)=\left[\begin{array}{c}
\int_{t-h}^{t} u_{1}\left(\tau-T_{10}\right) \phi(t-\tau) \mathrm{d} \tau \\
\int_{t-h}^{t} u_{1}\left(\tau-\tau_{1}-T_{10}\right) \phi(t-\tau) \mathrm{d} \tau \\
\vdots \\
\int_{t-h}^{t} u_{1}\left(\tau-\left(Q_{1}-1\right) \tau_{1}-T_{10}\right) \phi(t-\tau) \mathrm{d} \tau \\
\vdots \\
\int_{t-h}^{t} u_{K}\left(\tau-T_{K 0}\right) \phi(t-\tau) \mathrm{d} \tau \\
\vdots \\
\int_{t-h}^{t} u_{K}\left(\tau-\left(Q_{K}-1\right) \tau_{K}-T_{K 0}\right) \phi(t-\tau) \mathrm{d} \tau
\end{array}\right]
$$

Vector $\widetilde{\boldsymbol{b}}$ consists of parameters for each function and can be expressed as

$$
\widetilde{\boldsymbol{b}}=\left[\begin{array}{c}
b_{10} \\
b_{11} \\
\vdots \\
b_{1 Q_{1}-1} \\
\vdots \\
b_{K 0} \\
\vdots \\
b_{K Q_{K}-1}
\end{array}\right]
$$




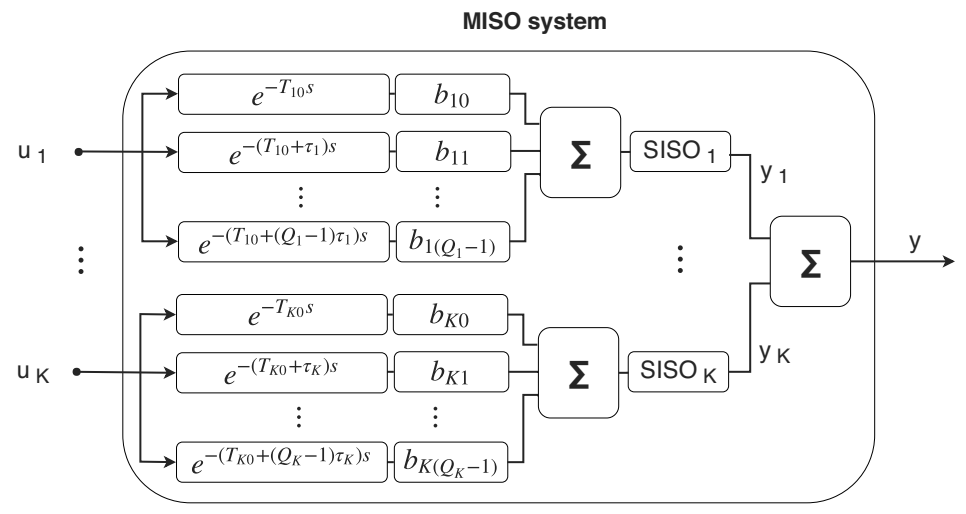

Fig. 2. MISO model diagram with delays.

The number of identified parameters in this case is equal to $n+\sum_{k=1}^{K} Q_{k}+1$. Further stages of the identification procedure are analogous as previously.

The described approach is valid on the condition that the real system input function can always be precisely estimated as a linear combination of the defined number of time-shifted input signals. The proof can be found in the work by Balestrino et al. (2000b).

\section{Decomposition idea for adaptive identification of the MISO model}

The main objective of this paper is to present a new idea of using the MFM for some nonlinear dynamic systems. The common use of the MFM for nonlinear systems is mostly adequate for the systems in the form

$$
y^{(n)}(t)+f_{n-1}^{(n-1)}[y(t)]+\cdots+f_{0}[y(t)]=b_{0} u(t) .
$$

Signals $y(t)$ and $u(t)$ are measured and all nonlinear functions $f_{i}[y(t)]$ are also known. The derivatives of $y^{(i)}(t)$ as well as $f_{i}^{(i)}[y(t)]$ are unknown. Then the MFM may be used directly. However, if the nonlinearity has the form $f_{i}\left[y^{(i)}(t)\right]$, then the MFM cannot be used.

The proposed modification of standard identification algorithms assumes the use of local linear models which accurately reflect the real system behaviour in specified time intervals. This approach enables on-line identification of nonlinear objects.

The described on-line algorithm implements an adaptive method for the best identification of the MISO system by repetition of the identification procedure in successive moving windows with width $T$. The identification of parameters is always based on the input-output data from the last window. Based on the system model obtained, the simulation procedure predicts the output for the next window (next interval). Then comparison between the simulated and the real outputs will be made in this window. The adaptive identification procedure includes two cases:

- If the simulated output concerning the last window is close enough to the real system output, then the procedure does not change the last model and continues the simulation for the next interval.

- If the simulation shows significant differences between the predicted and actual outputs, calculated based on the last window, then a new identification step is performed and the new model for this last data window is being searched.

An important idea used in this algorithm is that the parameter identification procedure for the MISO model is performed by the consecutive identification of the $\mathrm{SISO}_{k}$ models separately, for $k \in\{1, \ldots, K\}$. The classical approach for MISO systems (6) described in Section 3 is performed only once at the initial moment, to obtain the initial system parameters.

For each $\mathrm{SISO}_{k}$ model (7) the EEM for identification of parameters $\boldsymbol{\theta}_{k}$ is used with a suitably the chosen vector $\boldsymbol{\eta}_{k}$ corresponding to the parameters constraint $\boldsymbol{\eta}_{k} \boldsymbol{\theta}_{k}=1$. This identification of the single $\mathrm{SISO}_{k}$ model is performed in the interval $\left[t_{j-1}, t_{j}\right]=\left[t_{j}-T, t_{j}\right]$. In the next interval $\left[t_{j}, t_{j+1}\right]$, the outputs $y_{k}(t)$ of all $\mathrm{SISO}_{k}$ models are simulated and thus the full predicted output $y_{s}(t)=$ $\sum_{k=1}^{K} y_{k}(t)$ of the MISO model. In this interval the difference between the real system output $y(t)$ and $y_{s}(t)$ is found and the integrated square error is finally calculated using the OEM. If in the interval $\left[t_{j}, t_{j+1}\right]$ the output error of the full MISO model is greater than some prescribed value, then the identification procedure is repeated for the subsequent $\mathrm{SISO}_{k}$ models. A suitable system input and the hypothetical output $y_{k}(t)$, calculated as the difference between the real system output and the simulated outputs of other SISO models are used for the identification. In the next interval $\left[t_{j+1}, t_{j+2}\right]$ the obtained models parameters 
are applied. If in the interval $\left[t_{j}, t_{j+1}\right]$ the integral of the output square error of the full MISO model is less than some prescribed value, then the on-line identification procedure is omitted and for simulation of the MISO model output, the last obtained model is kept unchanged.

Hence, the main identification procedure can be interpreted as a nonlinear optimization algorithm of the Gauss-Seidel type (Khoury and Harder, 2016), where the main performance index of identification for the MISO model is based on the output error in the full space of all MISO parameters. The consecutive minimization steps in each subspace (orthogonal directions) associated with each $\mathrm{SISO}_{k}$ model are based on the EEM and local iterations of different $\boldsymbol{\eta}_{k}$. An idea similar to some extent, in which the MIMO system was divided into separately identified SISO systems, was described by Rao et al. (1984)

The second objective of this paper is the practical implementation of the above described identification approach for the problem of modeling glass temperature changes for a single zone of glass forehearth, in which a local PID controller is employed and the gas-air mixture is dosed. The identified system is described using a linear model with two inputs: gas-air mixture pressure and molten glass temperature from the previous forehearth zone. The model parameters can be changed during the on-line identification process to ensure a better fit for the current process characteristics, which is nonlinear. The method described by Byrski and Byrski (2012) serves as a basic part of the proposed algorithm. In this paper, this algorithm is also used for the extended version of the method, in which the MISO model is composed of multiple linear SISO models with time-shifted input signals. Including many systems with different time-delays has the objective of identifying the actual system time-delay value. Balestrino et al. (2000b) tested a similar approach.

\section{Temperature control for multi-zone glass forehearths}

The glassware manufacturing process can be divided into three main stages:

- melting raw materials in a glass furnace,

- stabilizing molten glass temperature in working end and forehearths zones,

- forming glass containers with forming machines.

The whole process is very energy-consuming. Significant amounts of gas or oil are used during the production not only to melt the glass batch, but also to heat molten glass flowing through the forehearths. Another aspect connected with production of glass containers concerns ensuring adequate quality of final product. Especially glass homogeneity, which depends on temperature differences between glass layers in the forehearth, has a big impact on this issue. During the technological process the type of the final product can be changed. In this situation a new operating point should be set, and the forehearth pull rate (weight of produced glass) and the temperature set points for regulators need to be changed. The production cannot be continued until the installation parameters are stabilized. An ideal control system should ensure quick operation, in order to minimize production downtimes. The single forehearth is divided into few zones, e.g., rear, front and conditioning as presented by Gough and Matovich (1997). In a typical case, each zone has its own temperature controller which controls gas-air mixture pressure. Some zones can also have installed other devices, like waste dampers for discharging exhaust gases and cooling dampers to lower the molten glass temperature.

As was already pointed out, the forehearth control system should ensure obtaining the desired temperature profile for the molten glass. The desired glass temperature in each zone depends on the specified product. It is measured with single or multi-point thermocouples. In the last zone, often three three-point thermocouples are installed. It allows determining the molten glass temperature for the whole profile. Based on these measures, glass homogeneity can be calculated. In the analyzed installation, each zone has its own temperature controller adjusting mixture valves positions (one for each zone). The diagram of the described forehearth control system is presented in Fig. 3 .

As was noticed previously, high demands on the described control system imply that conventional single control loops can be often insufficient for the analyzed problem. The proportional-integral-derivative (PID) controllers, which are primarily used, do not have a

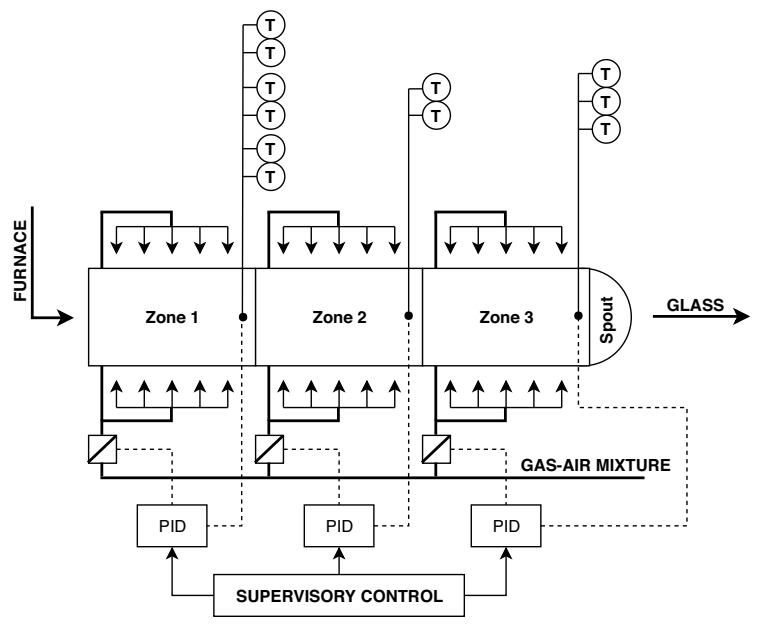

Fig. 3. Forehearth control system. 
predictive capability. Some authors proposed more sophisticated control structures. Ayla and Solis (1991) suggested replacing PID loops with a self-organizing fuzzy controller. Yet another idea involves using a predictive controller. Wang et al. (1997) claim that replacing PID controllers by the generalized predictive control (GPC) algorithm can improve the real process control quality. An interesting example of the predictive controller cooperating with an adaptive model was described by Gough and Matovich (1997). The problem of process model synthesis for a predictive controller was discussed by Grega et al. (2015). The finite element method was proposed to make the synthesis of the process model possible to use in a real-time environment. Grega et al. (2016) analyzed practical aspects of various variants of implementing the hierarchical controller for the forehearth control system. Advantages and drawbacks of the described solutions were pointed out.

\section{Algorithm description}

The method described in Section 4 is supposed to allow on-line identification of the single glass forehearth zone dynamics. The gas-air mixture pressure, which is the PID control variable, and molten glass temperature from the previous forehearth zone are treated as the model inputs, while the current zone glass temperature is the system output. The constructed model should allow predicting molten glass temperature changes for the current zone in response to the defined inputs for a short defined time horizon, which can be useful for on-line controller tuning.

The algorithm is based on the MFM, but as was pointed out in Section 4, this simple approach is not sufficient in this case. Due to the real plant nonlinearity, and the spatiotemporal nature, accurate simulation results cannot be obtained using an LTI model, for the whole time interval. It was adopted that the object parameters can change significantly during the identification. Finding suitable intervals, in which the model synthesis can be performed, is a key element of the procedure. Before a detailed method description, some assumptions have to be made:

- The model is linearized around a selected steady state.

- The model has a MISO form. Its output can be treated as a sum of individual SISO models outputs.

- Parameters of constitutive SISO models can be changed during the identification process, as well as the structure of the model (different number of delays for each SISO model control signal).

- The identification range is partitioned into smaller intervals. In the first interval of width $T_{\text {start }}$ only the whole MISO system identification is performed. In the other intervals of width $T$ the system simulation is performed and the model parameters can be changed by identifying individual SISO systems.

- There are transport delays for the model inputs which have to be included during the model synthesis - the measured signals are appropriately shifted.

6.1. Exact state observers. For the modeling and identification of the continuous $\mathrm{SISO}_{k}$ LTI subsystem of the MISO system presented in Fig. 1, the standard transfer function is used

$$
G_{k}(s)=\frac{b_{k n-1} s^{n-1}+\cdots+b_{k 0}}{a_{k n} s^{n}+\cdots+a_{k 1} s+a_{k 0}},
$$

where, by definition, the initial conditions are zero and no transport delay occurs.

Although the presented identification method with the use of modulating functions is insensitive to system initial conditions, the knowledge of these conditions is crucial for the output simulation purposes. Due to the above-mentioned model changes in consecutive time intervals, the ability to perform continuous simulation with nonzero initial conditions in each interval is essential. Hence, for the exact simulation procedure, besides of the transfer function of type 27, also the standard state space model will be used with suitable matrices:

$$
\begin{aligned}
& \dot{x}_{k}(t)=\boldsymbol{A} x_{k}(t)+\boldsymbol{B} u_{k}(t), \\
& y_{k}(t)=\boldsymbol{C} x_{k}(t)+\boldsymbol{D} u_{k}(t),
\end{aligned}
$$

where

$$
\begin{gathered}
x_{k}(t) \in \mathbb{R}^{n}, \quad u_{k}(t) \in \mathbb{R}, \quad y_{k}(t) \in \mathbb{R}, \quad \forall t>0, \\
\boldsymbol{A}=\left[\begin{array}{cccc}
0 & \ldots & 0 & -\frac{a_{k 0}}{a_{k n}} \\
1 & \ddots & \vdots & \vdots \\
\vdots & \ddots & 0 & -\frac{a_{k n-2}}{a_{k n}} \\
0 & \ldots & 1 & -\frac{a_{k n-1}}{a_{k n}}
\end{array}\right], \quad \boldsymbol{B}=\left[\begin{array}{c}
\frac{b_{k 0}}{a_{k n}} \\
\vdots \\
\frac{b_{k n-1}}{a_{k n}}
\end{array}\right], \\
\boldsymbol{C}=\left[\begin{array}{lll}
0 & \ldots & 1
\end{array}\right], \quad \boldsymbol{D}=0 .
\end{gathered}
$$

These matrices will guarantee the state observability, and hence, the use of the state observers. The standard asymptotic state observers, like the Luenberger observer, generate only the estimate of the actual state, which in a long interval asymptotically converges to the actual state. However, it is impossible to calculate exactly the estimation error at any moment, because the value of the actual state is unknown. Instead of this type of the differential state estimators, in the paper the exact state observers will be used. From the theory of exact state integral observers (Byrski and Byrski, 2016) it is known 
that such observers, for any final observation interval $T$ (assumed in advance), can calculate the actual value of the observed state $x_{k}\left(t_{1}\right)$ at the final moment $t_{1}$ of the observation window $\left[t_{1}-T, t_{1}\right]$. For the simulation and prediction of the state in the next time interval $\left[t_{1}, t_{1}+T\right]$ and hence for simulation of the unmeasured individual outputs $y_{k}(t)$ of the $\mathrm{SISO}_{k}$ models in this interval, one needs to know the exact value of the state $x_{k}\left(t_{1}\right)$ at the beginning of this interval. The final value from the previous interval will be used as the initial state condition in the next interval.

Below the general formula for the exact state observer of the final state $x_{k}(T) \in \mathbb{R}^{n}$ of the $\mathrm{SISO}_{k}$ system calculated in some general interval $[0, T]$ will be recalled. The final value of the state, at the moment $T$, is given by

$$
x_{k}(T)=\int_{0}^{T} \boldsymbol{G}_{\mathbf{1}}(t) y_{k}(t) \mathrm{d} t+\int_{0}^{T} \boldsymbol{G}_{\mathbf{2}}(t) u_{k}(t) \mathrm{d} t .
$$

Remark 3. In this section $\boldsymbol{G}_{\mathbf{1}}(t)$ and $\boldsymbol{G}_{\mathbf{2}}(t)$ denote the observer matrices.

One can see that after multiplication of matrix functions $\boldsymbol{G}_{\mathbf{1}}(t)$ by the output signal measurements $y_{k}(t)$ and matrix $\boldsymbol{G}_{\mathbf{2}}(t)$ by the input signal $u_{k}(t)$ on interval $[0, T]$ and integration of these products, the observer will give the exact state $x_{k}(T)$ for any chosen $T$. The form of these matrices as well as the form of the corresponding Gramian $M_{0}$ results from the definition of state observability. The observer matrices given for this basic interval $t \in[0, T]$ are

$$
\begin{gathered}
\boldsymbol{G}_{\mathbf{1}}(t)=e^{\boldsymbol{A} T} \boldsymbol{M}_{\mathbf{0}}^{-1} e^{\boldsymbol{A}^{\prime} t} \boldsymbol{C}^{\prime} \\
\boldsymbol{G}_{\mathbf{2}}(t)=e^{\boldsymbol{A} T} \boldsymbol{M}_{\mathbf{0}}^{-1}\left[\int_{0}^{t} e^{\boldsymbol{A}^{\prime} \tau} \boldsymbol{C}^{\prime} \boldsymbol{C} e^{\boldsymbol{A} \tau} \mathrm{d} \tau\right] e^{-\boldsymbol{A} t} \boldsymbol{B},
\end{gathered}
$$

where

$$
\boldsymbol{M}_{\mathbf{0}}=\int_{0}^{T} e^{\boldsymbol{A}^{\prime} \tau} \boldsymbol{C}^{\prime} \boldsymbol{C} e^{\boldsymbol{A} \tau} \mathrm{d} \tau .
$$

These matrices can be obtained from the general output equation of the system 29,

$$
y_{k}(t)=\boldsymbol{C} e^{-\boldsymbol{A}(T-t)} x_{k}(T)-\boldsymbol{C} \int_{t}^{T} e^{\boldsymbol{A}(t-s)} \boldsymbol{B} u_{k}(s) \mathrm{d} s .
$$

For calculation of $x(T)$ one should multiply both the sides of this equation by the matrix $e^{-\boldsymbol{A}^{\prime}(T-t)} \boldsymbol{C}^{\prime}$ and integrate it over $[0, T]$. For an observable system, the Gram matrix $M_{0}$ is nonsingular for any $T$. Then one can derive the form (30) of the observer and the matrices (31) and (32). Based on the formula (30) it is easy to see that the equation describing the shifted version of the integral observer, working in consecutive time intervals $\left[t_{p}-T, t_{p}\right]$ has receding limits

$$
\begin{aligned}
x_{k}\left(t_{p}\right) & =\int_{t_{p}-T}^{t_{p}} \boldsymbol{G}_{\mathbf{1}}\left(T-t_{p}+t\right) y_{k}(t) \mathrm{d} t \\
& +\int_{t_{p}-T}^{t_{p}} \boldsymbol{G}_{\mathbf{2}}\left(T-t_{p}+t\right) u_{k}(t) \mathrm{d} t,
\end{aligned}
$$

where the successive time moments are

$$
t_{p}=t_{0}+T_{\text {start }}+(p-1) \cdot T, \quad p=2,3, \ldots
$$

This type of the state observer will be used for the reconstruction of each $\mathrm{SISO}_{k}$ model state. The whole MISO system (37) state $x\left(t_{p}\right) \in \mathbb{R}^{K \cdot n}$ consists of the state vectors for all SISO submodels. After reconstruction of the final state $x\left(t_{p}\right)$ in the $p$-th interval, this state will be used as the initial condition for the simulation of the state in the next, $(p+1)$-th interval, and thus the calculation of all individual output signals $y_{k}$ of each $\mathrm{SISO}_{k}$ model can be performed.

Finally, the calculation of the whole simulated output function $y_{s}$ is possible, as a sum of individual $y_{k}$, which enables its comparison with the actual output $y$ measured in the $(p+1)$ interval.

There is one more important and interesting problem in the theory and application of exact state observers. It is the choice of the state observation interval $T$. In this paper, the time $T$ is the width of the observation window and also the width of the simulation intervals. For different $T$, the formulas (30) and (31) give different form of the exact state observers. Different state observers have different norms defined in the function space $\left(L^{2}[0, T]\right) \times$ $\left(L^{2}[0, T]\right)$

$$
\left\|\left(\boldsymbol{G}_{\mathbf{1}}, \boldsymbol{G}_{\mathbf{2}}\right)\right\|^{2}=\int_{0}^{T}\left[\sum_{i=1}^{n}\left(g_{1}^{i}(\tau)^{2}+\sum_{i=1}^{n}\left(g_{2}^{i}(\tau)^{2}\right)\right] \mathrm{d} \tau,\right.
$$

where $g_{1}^{i}(\tau), g_{2}^{i}(\tau)$ are the $i$-th row entries of vectors $G_{1}, G_{2}$.

Theoretically, from the point of view of observation problems, if in the input-output measurement the disturbances (noise) do not exist, then the exactness of state reconstruction does not depend on time $T$. However, if in measurements of $y$ and $u$ a disturbance occurs (bounded signals belonging to the unit ball $\left\|z_{1}\right\| \leq 1$, $\left\|z_{2}\right\| \leq 1$ ) then the reconstruction error will appear and its norm will be estimated by the observer norm

$$
\sqrt{0.5} \max _{\left(z_{1}, z_{2}\right)}\|\epsilon\|_{\mathbb{R}^{n}} \leq\left\|\left(\boldsymbol{G}_{\mathbf{1}}, \boldsymbol{G}_{\mathbf{2}}\right)\right\|_{L^{2} \times L^{2}} .
$$

It turns out that with decreasing $T$, the observer's norm increases, even up to infinity as $T$ tends to zero. Hence, in practice for disturbed measurements of $y$ and 
$u$ the reconstruction error (estimated by the norm of the observer) will be lower for higher $T$. Therefore the choice of the best $T$ is very important and will be the aim of the future research. By different exact state observers it is also possible to perform the reconstruction of the norm of different disturbances acting in the system (Byrski and Byrski, 2018).

6.2. Identification procedure description. The state equations of the MISO system, shown in Fig. 1, can be expressed in the form (37), where $K$ is the number of system inputs, $n$ signifies the degree of the transfer function denominator for the $k$-th input (it is assumed that denominators for each input have the same rank), $m_{k}$ means the degree of the transfer function numerator for the $k$-th input. It is assumed that for specified input $b_{k i}=0$ for $i>m_{k}$.

Applying the classical methodology based on the EEM, according to Section 3, for the whole analyzed system, did not give satisfactory results. The difference between the actual system output and the obtained simulation results was significant. Numerical optimization of the constraint vector $\boldsymbol{\eta}$ for stored historical data and the use of the general EEM for the whole MISO system, invariably produced the MISO model parameters that did not guarantee a good matching of the actual output values with the model response. Hence, one can conclude, that it is impossible to find one and best constraint vector $\boldsymbol{\eta}$ with the use of some nonlinear optimization procedure (e.g., the MATLAB fminsearch algorithm) for the whole MISO system. For this reason a new adaptive methodology inspired by the Gauss-Seidel method was adopted for the problem of finding the best fit of actual and simulated signals. The problem was decomposed into subproblems solved in subspaces with fewer dimensions.

In further research, the assumption of the transport multi-delayed model structure and the zero order of the transfer function numerator $m=0$, according to (22), turns out to be more appropriate than the system without delays and with the order $m=n-1$, like in the transfer function (27). Hence, according to Section 3.1, for each individual $\mathrm{SISO}_{k}$ transfer function, the model with $Q_{k}$ different delays given as

$$
\begin{aligned}
G_{k}(s)= & \frac{b_{k 0} e^{-T_{k 0} s}+b_{k 1} e^{-\left(T_{k 0+\tau_{k}}\right) s}+\ldots}{a_{k n} s^{n}+\cdots+a_{k 1} s+a_{k 0}} \\
& +\frac{b_{k\left(Q_{k}-1\right)} e^{-\left(T_{k 0}+\left(Q_{k}-1\right) \tau_{k}\right) s}}{a_{k n} s^{n}+\cdots+a_{k 1} s+a_{k 0}}
\end{aligned}
$$

will be assumed. The values of the corresponding parameters $b_{k i}$ are calculated during the identification procedure by the MFM, that is why some $b_{k i}=0$ are also possible to occur.

Hence, there are $K$ submodels. In the considered case of only a single delay for each $k$-th subsystem, the main problem is solved in the space $\mathbb{R}^{K \cdot(n+1)+K}$, while the space of the $k$-th subproblem has $n+2$ dimensions,

$$
\left.\begin{array}{l}
\mathbb{R}^{n+2} \rightarrow \text { EEM } \\
\vdots \\
\mathbb{R}^{n+2} \rightarrow \text { EEM }
\end{array}\right\} \rightarrow \text { OEM }
$$

In the case of additional inputs, assuming that each $k$-th input signal is associated with $Q_{k}$ time-shifted signals, the main problem is stated in the space $\mathbb{R}^{K \cdot(n+1)+\sum_{k=1}^{K} Q_{k}}$, while each subproblem space has $n+Q_{k}+1$ dimensions,

$$
\left.\begin{array}{l}
\mathbb{R}^{n+Q_{1}+1} \rightarrow \text { EEM } \\
\vdots \\
\mathbb{R}^{n+Q_{K}+1} \rightarrow \text { EEM }
\end{array}\right\} \rightarrow \text { OEM. }
$$

These subproblems are solved to find the best parameter vector $\boldsymbol{\theta}_{k}$ for $\mathrm{SISO}_{k}$ model according to the formula (20). At this stage, different constraint vectors $\boldsymbol{\eta}_{k}$ may be chosen. As was stated before by Byrski and Byrski (2012), it was proven that the vector guaranteeing the least value of the performance index 210 should be chosen as the eigenvector which corresponds to the minimal eigenvalue of the Gram matrix G. Numerical experiments for the real object indicate that, despite minimizing the performance index 21, the obtained parameters yield unsatisfactory simulation results for other data intervals. For this reason, the optimal vector $\boldsymbol{\eta}$ was found in subsequent iterations and simulations.

The performance index $E_{i}$ for the main problem is stated as the integrated square error of the difference between the actual system $y(t)$ and the simulated system output $y_{s}(t)(\mathrm{OEM})$ :

$$
E_{i}=\int_{t_{p}-T}^{t_{p}}\left(y(t)-y_{s}(t)\right)^{2} \mathrm{~d} t .
$$

The overall identification procedure can be divided into several steps. Its general outline is presented in the form of Algorithm 1 1 It should be noted that the presented algorithm can be used both off-line and on-line. In the first case, the collected historical data are used and forward simulation can be made at once for the whole interval. Otherwise, the prediction horizon is limited by the minimum delay of the uncontrolled input (e.g., the previous zone glass temperature in the described case).

The first stage of the procedure concerns finding the operating point, where the system can be linearized. It is clear that the derivatives of the state variables near the steady state should be zero. In the analyzed problem there is no direct information about the derivatives of the state variables. That is why the operating point is determined based on the convoluted output signals $y_{i}$. Their values should be smallest possible near this point. This idea is described in Algorithm 2 . 


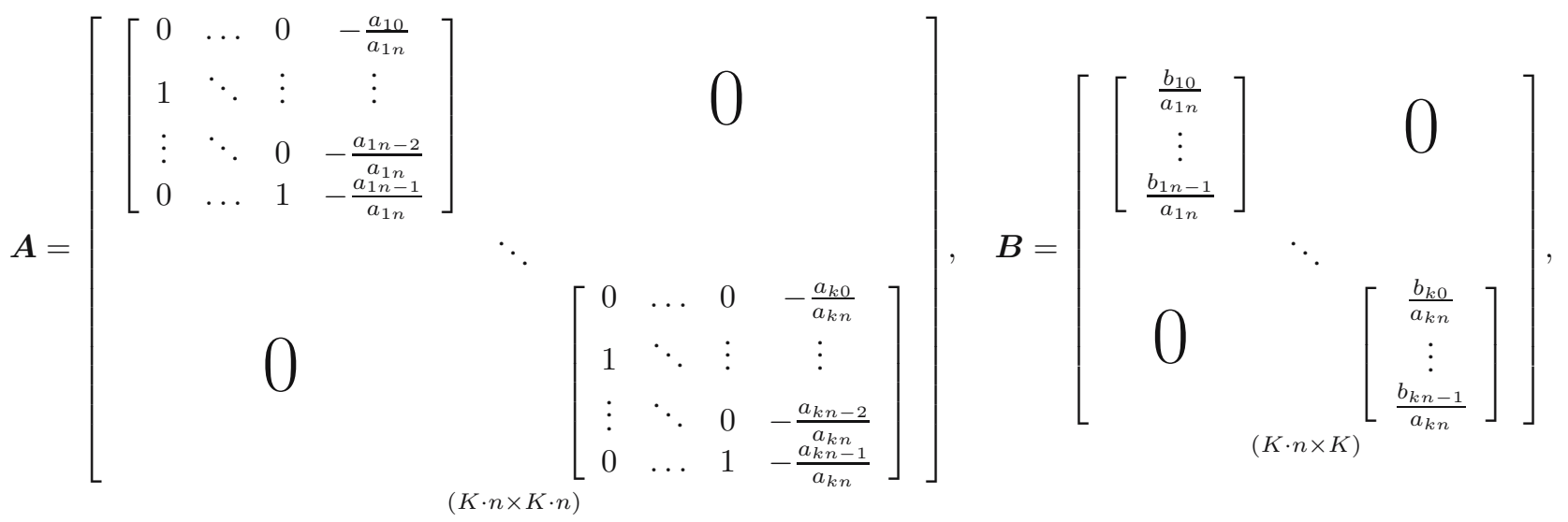

$$
\boldsymbol{C}=\left[\left[\begin{array}{lll}
0 & \ldots & 1
\end{array}\right] \underset{(1 \times K \cdot n)}{\ldots}\left[\begin{array}{lll}
0 & \ldots & 1
\end{array}\right]\right], \quad \boldsymbol{D}=\left[\begin{array}{lll}
0 & \ldots & 0
\end{array}\right]
$$

If the operating point is found, the specific identification procedure can be applied. As was mentioned before, model parameters are changed based on the input-output signal values only for the specified intervals. In the first interval, the MISO model is identified according to the algorithm described in Section 3 for systems with multiple inputs and a single output. The obtained MISO model can be presented as a sum of individual SISO models with the same denominators. These models are used for the system output simulation. In the next intervals, if the model output differs significantly from the actual system output, the re-identification is performed based on the data from the last interval. For each $k$-th input, the simulated $\mathrm{SISO}_{k}$ model output is obtained as the difference between the actual system output and the sum of simulated SISO models outputs different from $k$. Then, the $k$-th SISO model is identified using the registered input signal and the simulated output signal. The simulation for the whole MISO system is performed again for the updated system. If the new performance index (39) value is lower than for the previous model, the identification procedure is repeated for the next $(k+1)$-th SISO model. The SISO models updated previously are used for the simulation of the MISO system in the next steps. The whole operation for the single SISO systems is repeated until the performance index cannot be improved. Otherwise, the procedure is stopped and the best obtained models, minimizing the performance index (39) value, are stored. It is worth noting that the obtained SISO models may have different denominators, in contrast to the models obtained in the first interval. The described identification method is presented as Algorithm 3 .

\section{Experimental results}

In order to evaluate the effectiveness of the described method, based on the real process data, two experiments were performed off-line for previously collected historical data. In both of them, the same two data sets shown in Figs. 4 and 5 were used. The data were collected from the last forehearth zone (before the spout) of the real glass containers production line. The air-gas mixture is the only controlled process variable. All signals are sampled once per second. The molten glass temperatures can be measured in the range $900-1400{ }^{\circ} \mathrm{C}$ with thermocouples. Mixture pressure can be measured in the range $0-10$ $\mathrm{kPa}$. In practice, the maximum mixture pressure value that can be obtained is limited to $6 \mathrm{kPa}$. In both cases 32-bit analog-to-digital converters were used. Input and output signals for the system are measured directly, so the controller dynamics can be neglected in the analyzed problem.

It was assumed that the overall MISO model is composed of two submodels $(K=2)$ related to the system inputs. For the first submodel the mixture pressure is denoted as $u_{1}$ and the submodel output as $y_{1}$. Similarly, for the second SISO submodel the previous zone temperature is denoted as $u_{2}$ and the $\mathrm{SISO}_{2}$ output as $y_{2}$. In the first experiment, single delays were assumed for signals $u_{1}$ and $u_{2}$. One should substitute $Q_{1}=1$ and $Q_{2}=1$ into (24). In the second case, multiple time-shifted input signals were applied for the second signal: $Q_{1}=1, Q_{2}=5$. The MFM coefficients, common for both models, are presented in Table 1. In both experiments the same delay values for models were assumed: $13 \mathrm{~s}$ for $\mathrm{SISO}_{1}$ (the mixture pressure) and $50 \mathrm{~s}$ for $\mathrm{SISO}_{2}$ (the previous zone glass temperature). 
Algorithm 1. Overall identification procedure.

Step 1. Collect data for the input signals: $u_{1}(t), \ldots, u_{K}(t)$ where $k=1, \ldots, K$ as well as data for the single output signal $y(t)$. Specify the number of identification intervals $p_{\text {end }}$.

Step 2. Find the operating point time $t_{0}$ according to Algorithm 2 If $t_{0}<\infty$ perform the model linearization at this point and go to Step 3, otherwise go to Step 9.

Step 3. Perform the identification procedure based on the algorithm described in Section 3 starting from $t_{0}$ to $t_{0}+$ $T_{\text {start }}$ to obtain the MISO model parameters. Store suitable parameters for each $\mathrm{SISO}_{k}$ model.

Step 4. Perform simulation based on the obtained model for the first two intervals starting from $t_{0}$ to $t_{0}+T_{\text {start }}+T$, assuming zero initial condition. Start the state observer procedure and calculate $x\left(t_{0}+T_{\text {start }}+T\right)$. Store the simulated values of the state variables as the initial condition for the next interval $x_{0}=x\left(t_{0}+T_{\text {start }}+T\right)$. Set the interval counter $p=2$.

Step 5. Calculate the performance index in the current interval $E_{\text {curr }}$ according to 39 . If $E_{\text {curr }}$ is lower than a predefined threshold value $\operatorname{tr}_{2}$, do not change the current model. Otherwise, find the best model parameters starting from the $k$-th input for $k=1, \ldots, K$ as the initial model according to Algorithm 3 .

Step 6. Increment counter $p$. Perform forward simulation for the current interval with the initial condition $x_{0}$ starting from $x\left(t_{0}+T_{\text {start }}+T \cdot(p-2)\right)$ to $x\left(t_{0}+T_{\text {start }}+\right.$ $T \cdot(p-1))$.

Step 7. Update the model initial condition for the next interval $x_{0}=x\left(t_{0}+T_{\text {start }}+T \cdot(p-1)\right)$ using the state observer procedure.

Step 8. If $p<p_{\text {end }}$ return to Step 5, otherwise go to Step 9.

Step 9. Finish the identification procedure.

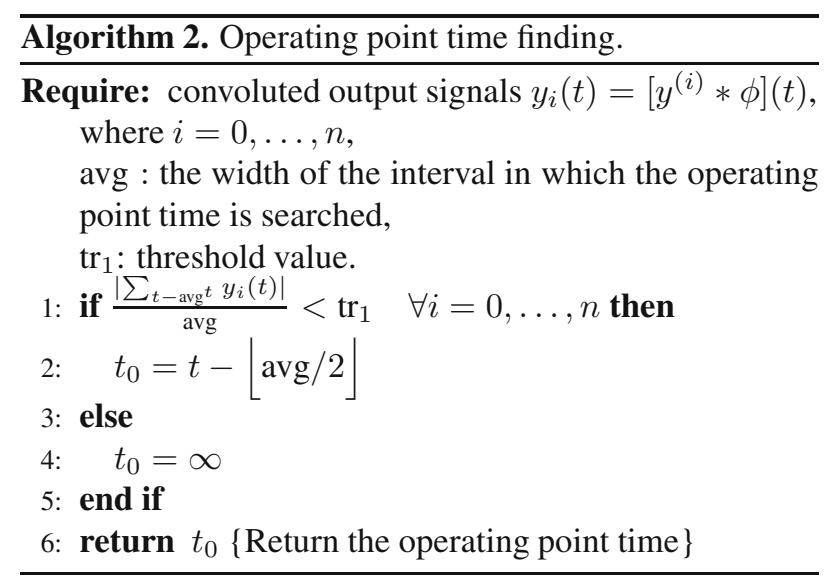

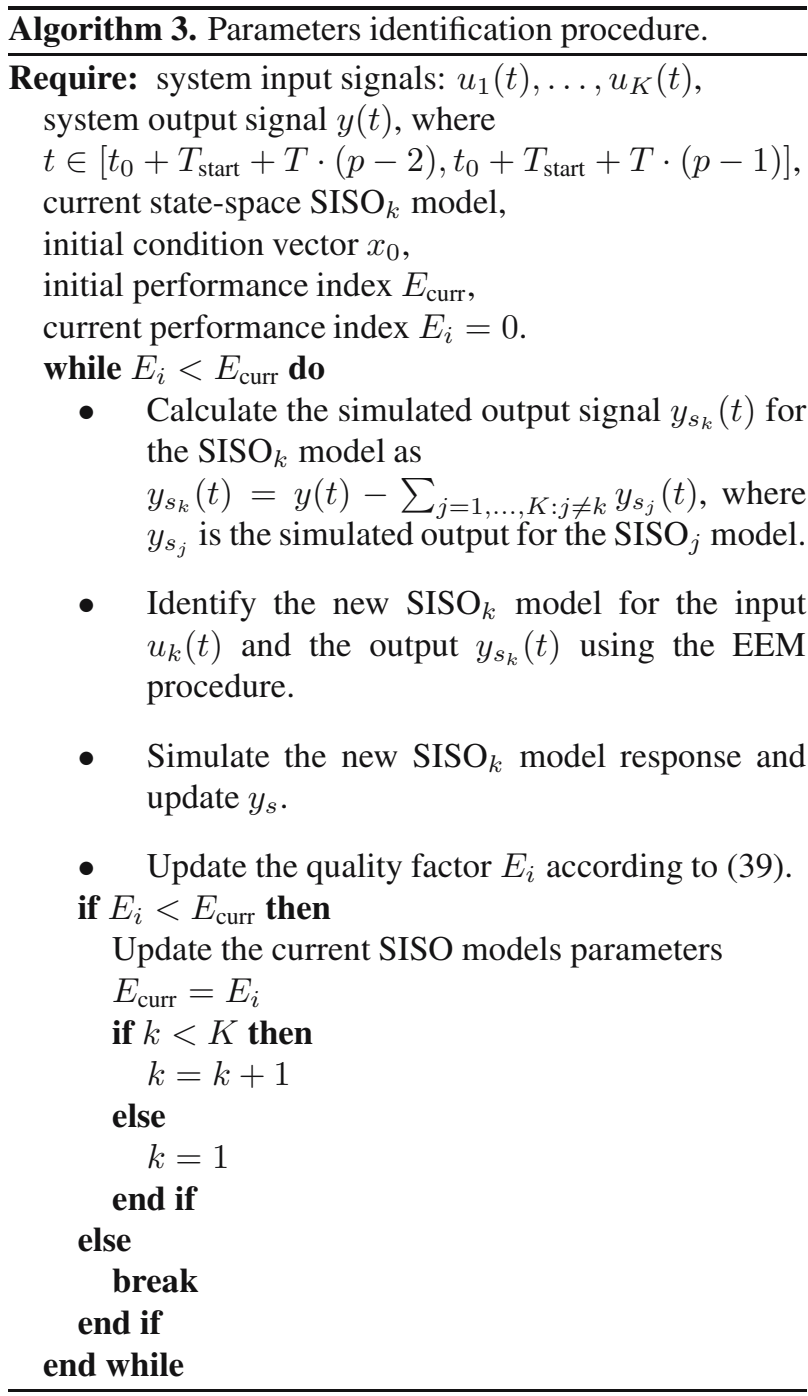

It should be noted that in the case of $Q_{k}=1$ there is only one time-shifted signal, while if $Q_{k} \neq 1$ this parameter corresponds to the central delay value. In both the cases local linear constraints were found. Elements of the vector $\boldsymbol{\eta}$ corresponding to the highest two powers of the transfer function denominator were smaller than the others, which allowed us to obtain larger values of the model parameters for these powers. Analogously the corresponding constraint $\boldsymbol{\eta}_{k}$ vector parameters for the identification of single $\mathrm{SISO}_{k}$ systems (7) have smaller values.

In Table 2 coefficients of the previously described model identification method are presented.

7.1. First experiment. The results of the first experiment are shown in Figs. 6 and 7 The intervals in which the changed models were applied for the system output prediction for the first time are marked as below:

- $B$ : the second interval in which both submodels 


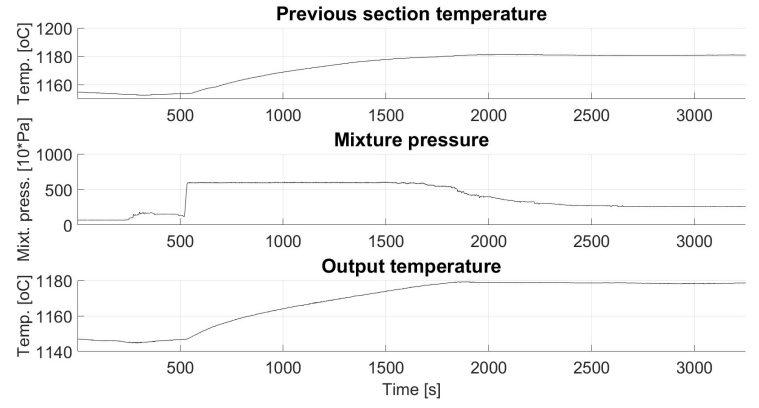

Fig. 4. Historical data: the first set. Two upper charts present the system inputs, while the third is the output.

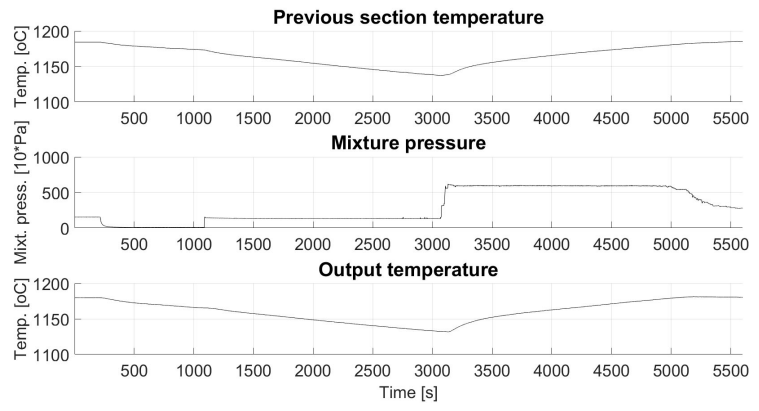

Fig. 5. Historical data: the second set. Two upper charts present the system inputs, while the third is the output.

parameters were changed according to the MISO system identification;

- $P$ : the interval in which the model parameters were changed starting with the $\mathrm{SISO}_{1}$ submodel;

- $T$ : the interval in which model parameters were changed starting with the $\mathrm{SISO}_{2}$ submodel.

The identified models parameters are presented in Tables $3 \sqrt{6}$ for the intervals in which they were applied. Noticeable changes in the parameter values can be seen during the simulation.

7.2. Second experiment. In the second experiment, the model parameters were identified with the use of multiple time-shifted input signals. Transport delay for the mixture pressure model can be specified accurately, while it is difficult to determine the delay between the previous zone glass temperature and the current system output. That is why four additional signals, shifted by $2 \mathrm{~s}$ forward and backward are considered as the model inputs. It made the overall number of the identified parameters increase up to 11 .

The experiment results are shown in Figs. 8 and 9 The intervals in which the model parameters were changed are described as in the previous case. The identified models parameters values are presented in
Tables 7f 12, For the second submodel (the glass temperature in the previous zone) parameters close to the assumed delay value have greater absolute values, which is consistent with predictions. Some of these parameters have negative values. A similar problem concerns the mixture pressure model for the second data set. In the intervals 9-14, its denominator value was negative. This was caused by very slight mixture pressure changes in these intervals connected with sudden changes of the measured output temperature. This can be counter-intuitive, however acceptable from the computational point of view, because each SISO model (even unstable) represents only theoretical dynamics on the short interval of the one control path whereas the whole MISO system is the sum of $K$ different SISO models. In this case one can also try to use an adaptation of the MFM by changing its parameters, as well as the identification interval $T_{I D}$. Then one can repeat the identification procedure for the last short interval. If this does not change the unstable model, then the obtained SISO model should not be used for the PID controller tuning.

7.3. Summary. The obtained results of two experiments for two data sets were compared. The mean integrated square error of the difference between the simulated output and the actual system output was adopted as a performance index in each case. The obtained results are presented in Table 13. The first experiment concerns the model with single time-shifted input signals, while the second is related to the use of multiple time-shifted input signals.

The results of both the performed experiments allow us to state that the obtained models gave sufficient approximation of the real system output. In each case both submodel parameters have changed along with the system

Table 1. MFM coefficients.

\begin{tabular}{|c|c|}
\multicolumn{2}{c}{ Table 1. MFM coefficients. } \\
\begin{tabular}{|c|c|}
\hline Parameter & Value \\
\hline \hline SISO $_{1}$ and SISO & model orders $n$ \\
Linear constraint vectors $\boldsymbol{\eta}, \boldsymbol{\eta}_{1}, \boldsymbol{\eta}_{2}$ & $1, \ldots, 0.15$, \\
& $0.15, \ldots, 1$ \\
Filtering function support width $h$ & $35 \mathrm{~s}$ \\
Loeb-Cahen functions parameters $N, M$ & 6,7 \\
\hline
\end{tabular}
\end{tabular}

Table 2. Identification procedure coefficients.

\begin{tabular}{|c|c|}
\hline Parameter & Value \\
\hline \hline Number of inputs $K$ & 2 \\
Operating point finding parameter 'avg' & 50 \\
Operating point threshold $\mathrm{tr}_{1}$ & $5 \times 10^{-2}$ \\
Model change threshold $\mathrm{tr}_{2}$ & 500 \\
First interval width $T_{\text {start }}$ & 500 \\
Identification interval width $T$ & 250 \\
\hline
\end{tabular}




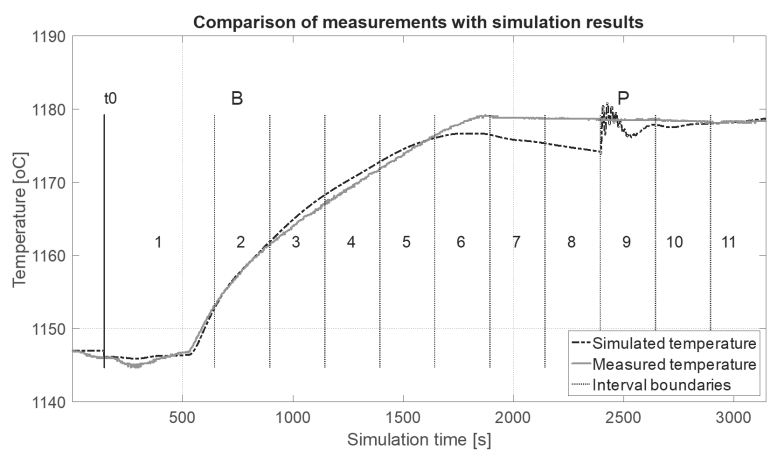

Fig. 6. Results for the first experiment (system with single time-shifted inputs): the first data set.

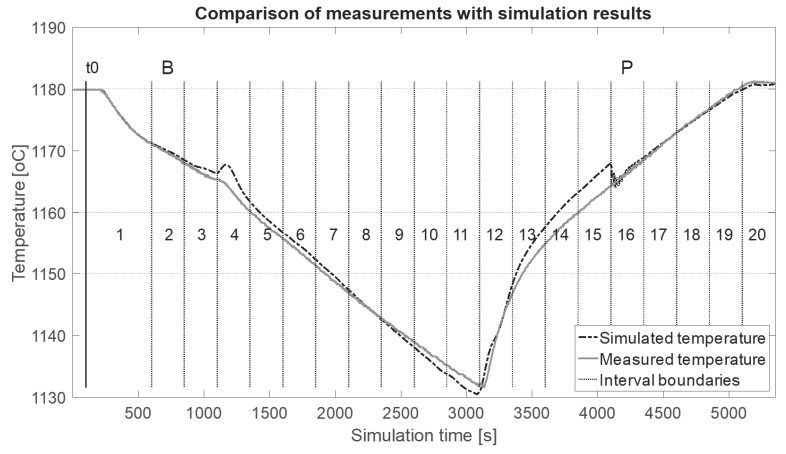

Fig. 7. Results for the first experiment (system with single time-shifted inputs): the second data set.

Table 3. Parameters values for $\mathrm{SISO}_{1}$ model $\left(u_{1} \rightarrow y_{1}\right)$ : the first experiment, the first data set (Fig. 4).

\begin{tabular}{|c|c|c|c|c|c|c|}
\hline \multirow{2}{*}{ Intervals } & \multicolumn{6}{|c|}{ Parameter } \\
\cline { 2 - 7 } & $a_{0}$ & $a_{1}$ & $a_{2}$ & $a_{3}$ & $a_{4}$ & $b_{0}$ \\
\hline \hline $1-8$ & $1.31 \times 10^{-4}$ & $8.55 \times 10^{-3}$ & 0.41 & 0.15 & 3.71 & $2.08 \times 10^{-4}$ \\
$9-11$ & $1.03 \times 10^{-5}$ & $8.71 \times 10^{-3}$ & 0.39 & 0.22 & 3.80 & $3.19 \times 10^{-5}$ \\
\hline
\end{tabular}

Table 4. Parameters values for $\mathrm{SISO}_{2}$ model $\left(u_{2} \rightarrow y_{2}\right)$ : the first experiment, the first data set (Fig. (4).

\begin{tabular}{|c|c|c|c|c|c|c|}
\hline \multirow{2}{*}{ Intervals } & \multicolumn{5}{|c|}{ Parameter } \\
\cline { 2 - 7 } & $a_{0}$ & $a_{1}$ & $a_{2}$ & $a_{3}$ & $a_{4}$ & $b_{0}$ \\
\hline \hline $1-8$ & $1.31 \times 10^{-4}$ & $8.55 \times 10^{-3}$ & 0.41 & 0.15 & 3.71 & $1.28 \times 10^{-4}$ \\
$9-11$ & $4.99 \times 10^{-5}$ & $1.07 \times 10^{-2}$ & 0.38 & 0.25 & 3.78 & $5.28 \times 10^{-5}$ \\
\hline
\end{tabular}

Table 5. Parameters values for $\mathrm{SISO}_{1}\left(u_{1} \rightarrow y_{1}\right)$ : the first experiment, the second data set (Fig. 5.

\begin{tabular}{|c|c|c|c|c|c|c|}
\hline \multirow{2}{*}{ Intervals } & \multicolumn{6}{|c|}{ Parameter } \\
\cline { 2 - 7 } & $a_{0}$ & $a_{1}$ & $a_{2}$ & $a_{3}$ & $a_{4}$ & $b_{0}$ \\
\hline \hline $1-15$ & $3.48 \times 10^{-4}$ & $1.31 \times 10^{-2}$ & 0.35 & 0.25 & 4.02 & $6.34 \times 10^{-4}$ \\
$16-20$ & $3.50 \times 10^{-4}$ & $1.43 \times 10^{-2}$ & 0.33 & 0.30 & 4.09 & $3.55 \times 10^{-4}$ \\
\hline
\end{tabular}

Table 6. Parameters values for $\mathrm{SISO}_{2}\left(u_{2} \rightarrow y_{2}\right)$ : the first experiment, the second data set (Fig. 5.

\begin{tabular}{|c|c|c|c|c|c|c|}
\hline \multirow{2}{*}{ Intervals } & \multicolumn{5}{|c|}{ Parameter } \\
\cline { 2 - 7 } & $a_{0}$ & $a_{1}$ & $a_{2}$ & $a_{3}$ & $a_{4}$ & $b_{0}$ \\
\hline \hline $1-15$ & $3.48 \times 10^{-4}$ & $1.31 \times 10^{-2}$ & 0.35 & 0.25 & 4.02 & $3.74 \times 10^{-4}$ \\
$16-20$ & $7.98 \times 10^{-5}$ & $1.51 \times 10^{-2}$ & 0.32 & 0.32 & 4.09 & $8.72 \times 10^{-5}$ \\
\hline
\end{tabular}




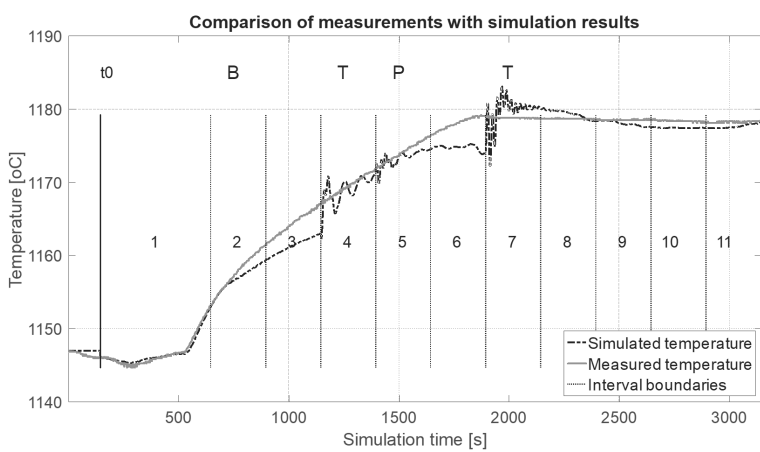

Fig. 8. Results for the second experiment (system with multiple time-shifted inputs): the first data set.

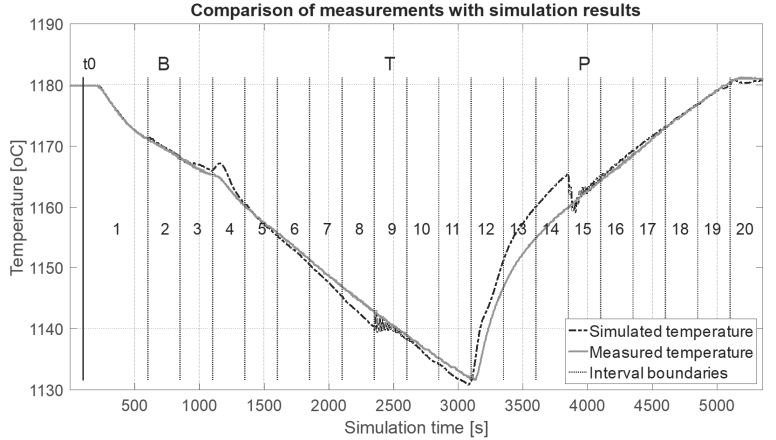

Fig. 9. Results for the second experiment (system with multiple time-shifted inputs): the second data set.

Table 7. Parameters values for $\mathrm{SISO}_{1}$ model $\left(u_{1} \rightarrow y_{1}\right)$ : the second experiment, the first data set (Fig. (4).

\begin{tabular}{|c|c|c|c|c|c|c|}
\hline \multirow{2}{*}{ Intervals } & \multicolumn{6}{|c|}{ Parameter } \\
\cline { 2 - 7 } & $a_{0}$ & $a_{1}$ & $a_{2}$ & $a_{3}$ & $a_{4}$ & $b_{0}$ \\
\hline \hline $1-3$ & $1.96 \times 10^{-4}$ & $9.79 \times 10^{-3}$ & 0.41 & 0.18 & 3.67 & $2.52 \times 10^{-4}$ \\
4 & $3.74 \times 10^{-5}$ & $1.64 \times 10^{-2}$ & 0.34 & 0.31 & 4.00 & $9.52 \times 10^{-5}$ \\
$5-6$ & $2.38 \times 10^{-3}$ & $7.16 \times 10^{-3}$ & 0.39 & 0.27 & 3.71 & $3.28 \times 10^{-3}$ \\
$7-11$ & $6.05 \times 10^{-5}$ & $6.89 \times 10^{-3}$ & 0.39 & 0.20 & 3.84 & $1.25 \times 10^{-4}$ \\
\hline
\end{tabular}

Table 8. Parameters $a$ values for $\mathrm{SISO}_{2}$ model $\left(u_{2} \rightarrow y_{2}\right)$ : the second experiment, the first data set (Fig. (4).

\begin{tabular}{|c|c|c|c|c|c|}
\hline \multirow{2}{*}{ Intervals } & \multicolumn{5}{|c|}{ Parameter } \\
\cline { 2 - 6 } & $a_{0}$ & $a_{1}$ & $a_{2}$ & $a_{3}$ & $a_{4}$ \\
\hline \hline $1-3$ & $1.96 \times 10^{-4}$ & $9.79 \times 10^{-3}$ & 0.41 & 0.18 & 3.67 \\
4 & $2.50 \times 10^{-3}$ & $7.13 \times 10^{-3}$ & 0.39 & 0.30 & 3.69 \\
$5-6$ & $2.67 \times 10^{-3}$ & $6.81 \times 10^{-3}$ & 0.40 & 0.24 & 3.66 \\
$7-11$ & $1.01 \times 10^{-3}$ & $5.39 \times 10^{-3}$ & 0.37 & 0.17 & 3.98 \\
\hline
\end{tabular}

Table 9. Parameters $b$ values for $\mathrm{SISO}_{2}$ model $\left(u_{2} \rightarrow y_{2}\right)$ : the second experiment, the first data set (Fig. 4).

\begin{tabular}{|c|c|c|c|c|c|}
\hline \multirow{2}{*}{ Intervals } & \multicolumn{5}{|c|}{ Parameter } \\
\cline { 2 - 6 } & $b_{0}$ & $b_{1}$ & $b_{2}$ & $b_{3}$ & $b_{4}$ \\
\hline \hline $1-3$ & $1.13 \times 10^{-2}$ & $-4.20 \times 10^{-2}$ & $5.37 \times 10^{-2}$ & $-2.32 \times 10^{-2}$ & $1.70 \times 10^{-4}$ \\
4 & $4.10 \times 10^{-2}$ & $-7.96 \times 10^{-2}$ & $5.48 \times 10^{-2}$ & $-1.94 \times 10^{-2}$ & $6.26 \times 10^{-3}$ \\
$5-6$ & $3.27 \times 10^{-2}$ & -0.10 & 0.14 & $-8.36 \times 10^{-2}$ & $2.10 \times 10^{-2}$ \\
$7-11$ & $9.86 \times 10^{-2}$ & -0.44 & 0.75 & -0.58 & 0.18 \\
\hline
\end{tabular}

Table 10. Parameters values for $\mathrm{SISO}_{1}$ model $\left(u_{1} \rightarrow y_{1}\right)$ : the second experiment, the second data set (Fig. 5).

\begin{tabular}{|c|c|c|c|c|c|c|}
\hline \multirow{2}{*}{ Intervals } & \multicolumn{6}{|c|}{ Parameter } \\
\cline { 2 - 7 } & $a_{0}$ & $a_{1}$ & $a_{2}$ & $a_{3}$ & $a_{4}$ & $b_{0}$ \\
\hline \hline $1-8$ & $4.93 \times 10^{-4}$ & $1.48 \times 10^{-2}$ & 0.35 & 0.29 & 3.91 & $7.49 \times 10^{-4}$ \\
$9-14$ & $6.97 \times 10^{-5}$ & $1.65 \times 10^{-2}$ & 0.36 & 0.27 & 3.90 & $-8.43 \times 10^{-4}$ \\
$15-20$ & $1.39 \times 10^{-3}$ & $1.56 \times 10^{-2}$ & 0.34 & 0.25 & 4.00 & $1.34 \times 10^{-3}$ \\
\hline
\end{tabular}

working conditions. Especially in the case of molten glass temperature significant changes in the previous forehearth zone, it can be observed that the model parameters are adjusted. These temperature fluctuations are often connected with a variable glass pull rate, which means that the mass of molten glass in the forehearth is changing. 
Table 11. Parameters $a$ values for $\mathrm{SISO}_{2}$ model $\left(u_{2} \rightarrow y_{2}\right)$ : the second experiment, the second data set (Fig. 5).

\begin{tabular}{|c|c|c|c|c|c|}
\hline \multirow{2}{*}{ Intervals } & \multicolumn{5}{|c|}{ Parameter } \\
\cline { 2 - 6 } & $a_{0}$ & $a_{1}$ & $a_{2}$ & $a_{3}$ & $a_{4}$ \\
\hline \hline $1-8$ & $4.93 \times 10^{-4}$ & $1.48 \times 10^{-2}$ & 0.35 & 0.29 & 3.91 \\
$9-14$ & $2.67 \times 10^{-4}$ & $1.52 \times 10^{-2}$ & 0.36 & 0.25 & 3.89 \\
$15-20$ & $5.55 \times 10^{-5}$ & $2.24 \times 10^{-2}$ & 0.27 & 0.56 & 4.18 \\
\hline
\end{tabular}

Table 12. Parameters $b$ values for $\mathrm{SISO}_{2}$ model $\left(u_{2} \rightarrow y_{2}\right)$ : the second experiment, the second data set (Fig. 5).

\begin{tabular}{|c|c|c|c|c|c|}
\hline \multirow{2}{*}{ Intervals } & \multicolumn{5}{|c|}{ Parameter } \\
\cline { 2 - 6 } & $b_{0}$ & $b_{1}$ & $b_{2}$ & $b_{3}$ & $b_{4}$ \\
\hline \hline $1-8$ & $3.28 \times 10^{-2}$ & $-9.33 \times 10^{-2}$ & $1.04 \times 10^{-1}$ & $-5.71 \times 10^{-2}$ & $1.42 \times 10^{-2}$ \\
$9-14$ & $8.72 \times 10^{-3}$ & $6.19 \times 10^{-3}$ & $5.41 \times 10^{-2}$ & $6.02 \times 10^{-2}$ & $2.07 \times 10^{-2}$ \\
$15-20$ & $-5.42 \times 10^{-2}$ & 0.18 & -0.22 & 0.12 & $-2.02 \times 10^{-3}$ \\
\hline
\end{tabular}

Table 13. Model identification results.

\begin{tabular}{|c|c|c|}
\hline \multirow{2}{*}{ Model input signals } & \multicolumn{2}{|c|}{ Output error } \\
\cline { 2 - 3 } & Data set 1 & Data set 2 \\
\hline \hline single time-shifted & 2.1032 & 1.9771 \\
multiple time-shifted & 2.1711 & 3.7148 \\
\hline
\end{tabular}

The current pull rate is not measured in the analyzed installation. It can be defined only in the case of stable working conditions, so the model parameters cannot be dependent on its value during the installation operating point changes. Some offset between the actual system trajectory and the simulated system output can be seen for both experiments, but the obtained accuracy is sufficient for control applications. This feature partly results from the applied state observation method. The simulated system output is used for obtaining the state estimate in each simulation interval, starting from the first one. This approach enables us to obtain a continuous simulated trajectory. However, the modeling errors may increase in the subsequent intervals.

Adopting additional time-shifted input signals in the second experiment did not improve the overall identification results, although the identified model parameters were adjusted more often and the optimal models were obtained noticeably earlier than in the first case. This methodology could be beneficial in the case of significant pull rate changes for the forehearth, because it is the main factor affecting the variable transport delay between the temperatures in the neighbouring forehearth zones. This delay was relatively small for the analyzed forehearth zone. This may result from the fact of installing glass stirrers in it. The aforementioned devices force a molten glass movement which results in faster mixing of liquids. Adopting additional time-shifted inputs could be reasonable for the other forehearth zones, which are significantly longer and are not equipped with the stirrers.

\section{Conclusions}

The performed experiments allow us to state that the proposed new method can be used for identification of the model parameters for the glass forehearth installation and makes it possible to predict the molten glass temperature changes for a short period of time. This allows quickly checking the PID controller parameters and, if necessary, their adaptive correction.

For both approaches (the first with single input signals delays and the second in which additional time-shifted signals were added) results sufficiently accurate to apply this method in the real control system were obtained. Significant modifications of the standard algorithm involving the MISO model decomposition to separate SISO models with cooperation of EEM and OEM gave satisfying results of identification. The mean integrated square error remained in the range of several percent in each experiment and the shape of the simulated temperature trajectory was close to the actual one. The models inaccuracies were noticeable in both the cases, especially for the second data set, but the proposed mechanism of updating the system parameters leads to keeping up with the actual object output over time. The models obtained with the use of the proposed optimization algorithm based on the Gauss-Seidel method can successfully reflect the glass forehearth temperature changes. Based on the presented research results, specialized software was developed, that will be used in the local controller tuning system in the technological line of the glass production process.

\section{Acknowledgment}

This work was partially supported with the scientific research funds from the Polish Ministry of Science and Higher Education and within the AGH UST grant no. 16.16.120.773, and was conducted within the research of EC Grant H2020-MSCA-RISE-2018/824046. 


\section{References}

Asiri, S.M. and Laleg-Kirati, T. (2017). Modulating functions-based method for parameters and source estimation in one-dimensional partial differential equations, Inverse Problems in Science and Engineering 25(8): 1191-1215.

Ayla, L. and Solis, J. (1991). Structured logic control in glass preparation processes, IEEE Transactions on Industry Applications 27(1): 108-111.

Balestrino, A., Landi, A. and Sani, L. (2000a). Identification of Hammerstein systems with input/output time delay via modulating functions, IFAC Proceedings Volumes 33(23): 199-203.

Balestrino, A., Landi, A. and Sani, L. (2000b). Parameter identification of continuous systems with multiple-input time delays via modulating functions, IEE Proceedings: Control Theory and Applications 147(1): 19-27.

Byrski, J. and Byrski, W. (2012). The role of parameter constraints in $\mathrm{EE}$ and $\mathrm{OE}$ methods for optimal identification of continuous LTI models, International Journal of Applied Mathematics and Computer Science 22(2): 379-388, DOI: 10.2478/v10006-012-0028-3.

Byrski, J. and Byrski, W. (2016). A double window state observer for detection and isolation of abrupt changes in parameters, International Journal of Applied Mathematics and Computer Science 26(3): 585-602, DOI: 10.1515/amcs-2016-0041.

Byrski, J. and Byrski, W. (2018). An optimal identification of the input-output disturbances in linear dynamic systems by the use of the exact observation of the state, Mathematical Problems in Engineering: 1-15, Article ID 8048567, DOI: 10.1155/2018/8048567.

Byrski, W. and Fuksa, S. (1995). Optimal identification of continuous systems in L2 space by the use of compact support filter, International Journal of Modelling \& Simulation 15(4): 125-131.

Byrski, W. and Kubiński, R. (1997). The convolution method for optimal identification generalized to MIMO continuous systems, Modelling, Identification and Control: Proceedings of the 16th IASTED International Conference, Innsbruck, Austria, pp. 44-47.

Cieza, O.B., Tafur, J.C. and Reger, J. (2014). Frequency domain modulating functions for continuous-time identification of linear and nonlinear systems, 16th Latinamerican Control Conference, At Quintana Roo, Mexico, pp. 690-695.

Co, T. and Ydstie, B. (1990). System identification using modulating functions and fast Fourier transforms, Computers \& Chemical Engineering 14(10): 1051-1066.

Gough, B.P. and Matovich, D. (1997). Predictive-adaptive temperature control of molten glass, IEEE Industry Applications Society Dynamic Modeling Control Applications for Industry Workshop, Vancouver, BC, Canada, pp. 51-55.

Grega, W., Piłat, A. and Tutaj, A. (2015). Modelling of the glass melting process for real-time implementation, International Journal of Modeling and Optimization 5(6): 366-373.
Grega, W., Tutaj, A., Klemiato, M. and Byrski, W. (2016). Comparison of real-time industrial process control solutions: Glass melting case study, 21st International Conference on Methods and Models in Automation and Robotics (MMAR), Międzyzdroje, Poland, pp. 122-127.

Janiczek, T. (2010). Generalization of the modulating functions method into the fractional differential equations, Bulletin of the Polish Academy of Sciences: Technical Sciences 58(4): 593-599.

Jouffroy, J. and Reger, J. (2015). Finite-time simultaneous parameter and state estimation using modulating functions, IEEE Conference on Control Applications (CCA), Sydney, NSW, Australia, pp. 394-399.

Khoury, R. and Harder, D. (2016). Numerical Methods and Modelling for Engineering, Springer, Cham.

Kozłowski, J. and Kowalczuk, Z. (2015). On-line parameter and delay estimation of continuous-time dynamic systems, International Journal of Applied Mathematics and Computer Science 25(2): 223-232, DOI: 10.1515/amcs-2015-0017.

Maletinsky, V. (1979). Identification of continuous dynamical systems with spline-type modulating functions method, IFAC Proceedings Volumes 12(8): 275-281.

Pearson, A., Shen, Y. and Klein, V. (1994). Application of Fourier modulating functions to parameter estimation of a multivariable linear differential system, IFAC Proceedings Volumes 27(8): 1013-1018.

Preisig, H. and Rippin, D. (1993). Theory and application of the modulating function method. I: Review and theory of the method and theory of the spline-type modulating functions, Computers \& Chemical Engineering 17(1): 1-16.

Rao, G.P., Diekmann, K. and Unbenhauen, H. (1984). Parameter estimation in large scale interconnected systems, IFAC Proceedings Volumes 17(2): 729-733.

Rao, G.P. and Sivakumar, G. (1979). Identification of deterministic time-lag systems, IEEE Transactions on Automatic Control 21(4): 527-529.

Rao, G.P. and Unbehauen, G. (2006). Identification of continous-time systems, IEE Proceedings: Control Theory and Applications 153(2): 185-220.

Shinbrot, M. (1957). On the analysis of linear and nonlinear systems, Transactions of the American Society of Mechanical Engineers: Journal of Basic Engineering 79: 547-552.

Wang, Q., Chalaye, G., Thomas, G. and Gilles, G. (1997). Predictive control of a glass process, Control Engineering Practice 5(2): 167-173.

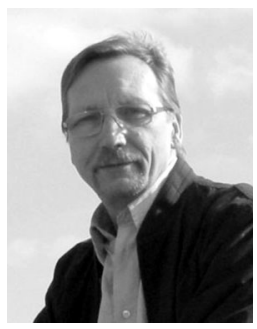

Witold Byrski received the DSc degree in 1994 and the professorial title in 2009 . He is employed as a full professor and manages the Group of Process Control at the Department of Automatic Control and Robotics (DACR), Faculty of Electrical Engineering, Automatics, Computer Science and Biomedical Engineering, AGH-UST in Kraków. His research covers identification of continuous dynamic models using modulating functions and convolution integrals, and new 
methods used for exact state observation based on integral operators. He has once been a visiting professor at Birmingham University, School of Electrical Engineering (GB), the Technical University of Denmark, Automation and Control Group, Lyngby (DEN), the Lille University of Science and Technology, Laboratory of Automatic Control (École Centrale Villeneuve-d'Ascq) (F), and the University of Groningen, Department of Mathematics (NL)

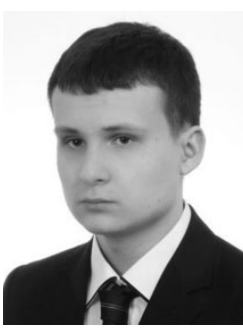

Michał Drapała obtained the MSc degree in automatic control and robotics in 2016 from the AGH University of Science and Technology in Kraków, Poland. Presently he is a PhD student in the Department of Automatic Control and Robotics, AGH-UST. His research interests include application of advanced control and identification methods for industrial processes. He is also a specialist in glass melting and conditioning technology, and a designer of control systems.

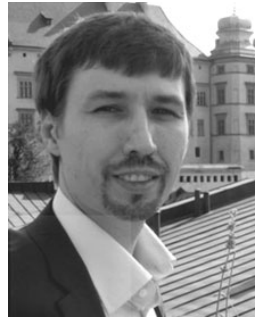

Jedrzej Byrski obtained the $\mathrm{PhD}$ degree in computer science in 2014. Presently he is employed as an assistant professor at the Department of Applied Computer Science, Faculty of Electrical Engineering, Automatics, Computer Science and Biomedical Engineering, AGH-UST in Kraków. His research interests cover mathematical algorithms for fault detection and identification in computer control systems, safety problems in computer networks and firewalls, the CORBA system, and real time computer systems in the QNX operating environment. He has participated in many scientific research projects within domestic grants (Polish Committee for Scientific Research, Ministry of Science and Higher Education) as well as European research projects. He is the head of the High Performance Computing Laboratory.

Received: 18 December 2018

Revised: 18 May 2019

Re-revised: 23 July 2019

Accepted: 18 August 2019 